\title{
Mineral inclusions as models to characterize deviatoric stress in single crystals
}

\author{
M. Morana ${ }^{1}$, R.J. Angel ${ }^{2}$, A. Girani ${ }^{1}$, M. Murri ${ }^{1}$, F. Alabarse ${ }^{3}$, M. Alvaro ${ }^{1}$ \\ ${ }^{1}$ Department of Earth and Environmental Sciences, University of Pavia, Via A. Ferrata 1, 27100, Pavia, Italy, \\ ${ }^{2}$ Istituto di Geoscienze e Georisorse, CNR, Via Giovanni Gradenigo, 6, 35131 Padua, Italy, \\ ${ }^{3}$ Elettra Sincrotrone Trieste ScPA, Strada Statale 14, km 163.5, Basovizza, 34149 Trieste, Italy
}

marta.morana01@universitadipavia.it

Non-hydrostatic stress is known to change the evolution of unit cell parameters [1] and the compression of bond lengths and angles in the structures of crystals, e.g. [2]. The resulting modifications in the structures can lead to changes in the physical and thermodynamic properties of crystals, and thus change their thermodynamic stability. As a consequence, both reconstructive phase transitions [3] and displacive-type symmetry-breaking phase transitions [4] under deviatoric stress can occur at different temperatures and different mean stress than under hydrostatic pressure. Despite its importance, the effect of non-hydrostatic stress on crystal structures is still poorly understood, because it is challenging to perform experiments under controlled deviatoric stress conditions. On the other hand, mineral host-inclusion systems composed of a mineral entrapped inside another mineral provide the perfect example to characterize a crystal under deviatoric stress. Because the inclusion is entrapped inside another crystal, it will not be under hydrostatic pressure and the deviatoric stress imposed on it will be the result of the difference in the elastic properties of the two crystals and their mutual crystallographic orientations. In this contribution, we describe a methodology to characterize the effect of deviatoric stress on inclusion crystal structures using synchrotron x-ray diffraction, including how to deal with the experimental challenges in the collection of intensity data from a host-inclusion system, and the evaluation of the quality of the results. The quartz in garnet system is an ideal candidate for this study. Quartz has a simple and well-known structure, whose variation with pressure and temperature has been widely characterised, while garnet, being cubic, imposes an almost isotropic strain on the inclusions, thus providing a relatively simple case study. Furthermore, quartz is one of the most common mineral inclusions in different types of rocks, so it qualifies as an interesting case for geological applications.

[1] Bassett, W. A. (2006). J. Phys.: Condens-Mat., 18(25), S921.

[2] Gatta, G. D., Kantor, I., Ballaran, T. B., Dubrovinsky, L., \& McCammon, C. (2007). Effect of non-hydrostatic conditions on the elastic behaviour of magnetite: an in situ single-crystal X-ray diffraction study. Phys, Chem. Miner., 34(9), 627-635.

[3] Richter B., Stünitz H. \& Heilbronner R. (2016) J. Geophys. Res. -Solid Earth, 121, 8015-8033.

[4] Bismayer U., Salje E. \& Joffrin C. (1982) J. Phys., 43, 1379-1388.

\section{Keywords: non-hydrostatic stress; $x$-ray diffraction; host-inclusion system; structure}

This work was supported by funding from the European Research Council under the European Union's Horizon 2020 research and innovation program grant agreement 714936 (ERC-STG TRUE DEPTHS) to Matteo Alvaro. It was also supported by the Ministero dell'Istruzione dell'Università e della Ricerca (MIUR)Progetti di Ricerca di Interesse Nazionale (PRIN), Bando PRIN 2017 - Prot. 2017ZE49E7_005 to Matteo Alvaro. We acknowledge Elettra Sincrotrone Trieste for providing access to synchrotron radiation beamtine at Xpress beamline (Proposal 20190101). 